\title{
Tevatron combined single top production cross sections
}

\author{
Manfredi Ronzani* (on behalf of the CDF and D0 collaborations) \\ Albert-Ludwigs-Universitaet Freiburg (DE) \\ E-mail: manfredi.ronzaniecern.ch
}

This paper reports the most recent measurements of the cross sections for the two main production modes of single top quarks in $p \bar{p}$ collisions at a center-of-mass energy of $1.96 \mathrm{TeV}$ in the Run II data collected with the CDF and D0 detector at the Fermilab Tevatron Collider. For this measurement the full data set corresponding to an integrated luminosity of up to $9.7 \mathrm{fb}^{-1}$ per experiment is used. We report the first observation of single top quark production in the $s$-channel with a significance of 6.3 standard deviations. We also present a simultaneous measurement of the production cross section of $t$ - and $s+t$-channels. Using these measurements we set a lower limit on the CKM matrix element $\left|\mathrm{V}_{\mathrm{tb}}\right|>0.92$ at 95\% C.L., assuming $m_{t}=172.5 \mathrm{GeV}$. Moreover, we present a search for charged massive resonances decaying to top ( $\mathrm{t}$ ) and bottom (b) quarks performed at CDF in the same data set. No significant excess above the standard model (SM) background prediction is observed and constraints on the $W^{\prime} \rightarrow t b$ mass and couplings in the 300 to $900 \mathrm{GeV} / c^{2}$ range are set.

The European Physical Society Conference on High Energy Physics 22-29 July 2015

Vienna, Austria

${ }^{*}$ Speaker. 


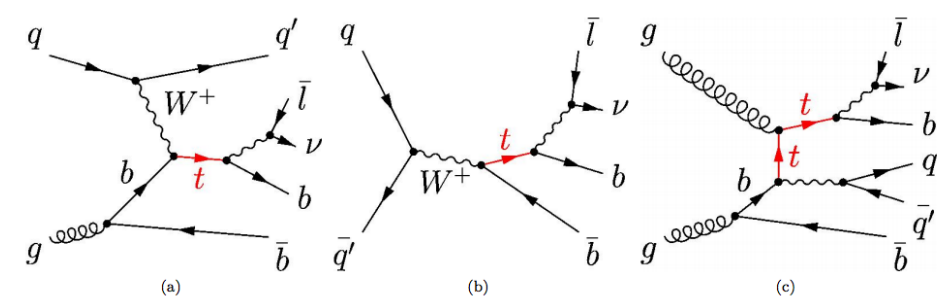

Figure 1: Single top quark production modes: (a) $t$-channel at NLO with initial state gluon splitting, (b) $s$-channel at leading order, and (c) $W t$-channel at NLO with initial state gluon splitting.

\section{Introduction}

The top quark was observed at the Tevatron by CDF [1] and D0 [2] in 1995 in $t \bar{t}$ pairs produced via strong interaction that is the top quark primary production mode. The Standard Model (SM) predicts top quark to be produced also singly via electroweak interaction (single top, ST) with three different production processes (Fig.1): $t$-channel with the exchange of a virtual $\mathrm{W}$ boson, $s$-channel with the $\mathrm{W}$ boson decaying into a top and an antibottom quarks and the $W t$-channel with the associate production of a $\mathrm{W}$ boson and a top quark. While the first two production modes have a small but measurable cross section at Tevatron (around $2 \mathrm{pb}$ and $1 \mathrm{pb}$, respectively) the Wt production mode has a cross section of $0.25 \mathrm{pb}$, that makes its contribution to the signal rate negligible [3].

Single top quark was first observed in 2009 by CDF [4] and D0 [5] experiments with an inclusive search in $s+t$ combined channels. The measurement in each single channel is done differently at the Tevatron and LHC, taking in account the differences in the production cross sections. The $t$-channel is the dominant process at both the Tevatron and LHC and it has been observed by D0 in 2011 [6] and then established by ATLAS [7] and CMS [8]. The $W t$-channel is visible only at the LHC and it has been observed by CMS collaboration at $8 \mathrm{TeV}$ [9]. The $s$-channel has a relatively small cross section at both Tevatron and LHC but at LHC the signal to background ratio is smaller, that gives an advantage for the observation to the Tevatron experiments.

The single top quark cross section measurement is proportional to $\left|\mathrm{V}_{\mathrm{tb}}\right|^{2}$, where $\left|\mathrm{V}_{\mathrm{tb}}\right|$ is element of the Cabibbo-Kobayashi-Maskawa (CKM) matrix. A direct measurement of $\left|\mathrm{V}_{\mathrm{tb}}\right|$ is therefore possible, that is also a test of the unitarity of the CKM matrix and it can constrain extensions of the SM, for example with fourth quark generation [10].

Several extension of the SM predicts the existence of massive, short-lived states decaying to pairs of SM leptons or quarks. Such a resonance decaying to a top (t) and a bottom (b) quark, shares the same final state as the single-top quark production. Recent searches in the $W^{\prime} \rightarrow t b$ channel have been performed by the CDF [11] and D0 [12] collaborations as well as by ATLAS [13] and CMS [14]. In the mass region well below $1 \mathrm{TeV}$ the Tevatron experiments have greater sensitivity due to the relative suppression of gluon-initiated backgrounds compared to the quark-initiated signals.

\section{Selection of Single Top Quark Events}

The SM predicts the top quark to decay almost always into a real $W$ boson and a $b$ quark. The 
further decay of the $W$ boson selects the final state. Both CDF and D0 use lepton+jets final state selection requiring the presence of an high- $\mathrm{p}_{\mathrm{T}}$ isolated lepton (electron or muon), large missing transverse energy $\left(\not_{T}\right)$ and 2 or 3 jets, at least one of them identified as coming from a b quark (1 or 2 b-tags). At CDF, the $\mathbb{E}_{T}+$ jets final state selection is also investigated. In the $\mathbb{E}_{T}+$ jets selection the products from the decay of the $\mathrm{W}$ boson are not reconstructed or they contain $\tau$ leptons which decay hadronically. This selection is orthogonal to the lepton+jets one and adds approximately $33 \%$ of acceptance.

Different background processes are considered: events with a $\mathrm{W}$ boson produced with one or more heavy flavor jets $(\mathrm{W}+\mathrm{HF})$ or with light-flavor jets that are mistagged as coming from $a b$ quark (W+LF), events from diboson processes (WW,ZZ,WZ), events with a $\mathrm{Z}$ boson and jets, $t \bar{t}$ pair production and multijets (non-W) events where a $\mathrm{W}$ boson is falsely reconstructed. All the background processes (except multijets) and the single top signal events are modeled using various MC event generators. Single top signals are modeled for a top quark mass of $m_{t}=172.5 \mathrm{GeV} / \mathrm{c}^{2}$ using POWHEG (CDF) [16] or COMPHEP (D0 )[15]. The multijet background is modeled using data. Different multivariate analysis methods (MVA) and combination of different MVAs are used to extract the signal from the background.

\section{CDF and Do Single Top production cross-section measurements}

\subsection{Do Single Top Analysis with $9.7 \mathrm{fb}^{-1}$}

The D0 single top analysis is performed in the combined $s+t$ sample using events selected in the lepton+jets final state. The tagging algorithm is based on a multivariate technique [17]. Three multivariate methods are used: matrix element (ME), Bayesian Neural Network (BNN) and boosted decision tree (BDT). The correlation among the output of the individual MVA methods is measured to be around 75\%. A final BNN is built in order to construct combined discriminants for $t$ - and $s$-channel signals ( $D_{t}^{\text {comb }}$ and $D_{s}^{\text {comb }}$ ), shown in Fig.2(a) and Fig.2(b), respectively. The inclusive $s+t$ single top cross section as well as the $t$ - and $s$-channel individual cross section are extracted using a Bayesian approach. With no assumptions on the relative $t$ - and $s$-channel contributions, the total single top cross section is measured to be $\sigma_{s+t}=4.11_{-0.55}^{+0.59} \mathrm{pb}[19]$. The measured cross section is $\sigma_{s}=1.10_{-0.31}^{+0.33} \mathrm{pb}$, corresponding to a significance of 3.7 standard deviations [19].

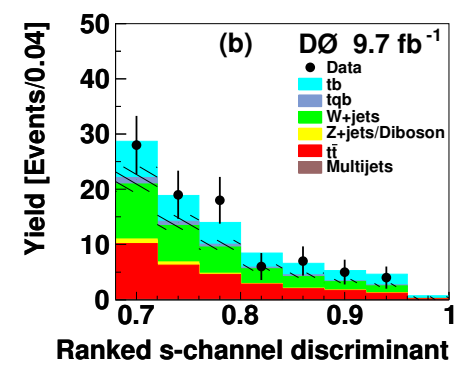

(a)

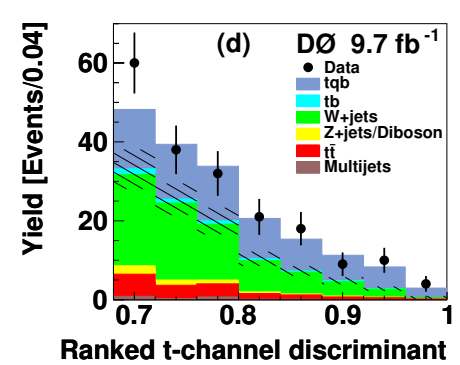

(b)

Figure 2: D0 lepton+jets discriminant distributions in the high discrimininant region shown for the $s$-channel (a) and $t$-channel (b). 


\subsection{CDF Single Top cross section measurements}

The CDF strategy has been oriented to perform different analyses for the inclusive $s+t$ and $s$-channel cross section measurement which have been fully investigated in the lepton+jets and $E_{T}+$ jets final states.

The lepton+jets analysis in the $s+t$ inclusive channel is performed in $7.5 \mathrm{fb}^{-1}$ of integrated luminosity. The analysis builds 4 independent signal regions (SRs) depending from the jet multiplicity ( 2 or 3 ) and the number of b-tags (1 or 2). A Neural Network (NN) discriminant (Fig. 3(a)) is built from a number of kinematic variables with good discriminating power [20]. The $t$ - and $W t$ - channels are combined as they share the same final state topology. The total single top cross section value is measured to be $\sigma_{\mathrm{s}+\mathrm{t}+\mathrm{Wt}}=3.04_{-0.53}^{+0.57} \mathrm{pb}$ for a top quark mass of $\mathrm{m}_{\mathrm{t}}=172.5 \mathrm{GeV} / \mathrm{c}^{2}$. The $E_{T}+$ jets analysis in the $s+t$ inclusive channel is performed in $9.5 \mathrm{fb}^{-1}$ of CDF integrated luminosity [21]. A signal $\mathrm{NN}_{s+t}^{\mathrm{sig}}$ (Fig. 3(b)) is used to separate both $s$ - ant $t$-channel signal processes from the background [21]. The results of the two CDF $s+t$ analyses (lepton+jets and $\not_{T}+$ jets) are combined. The combined $\sigma_{s+t}$ measurement results in a single top quark production cross section of $\sigma_{s+t}=3.02_{-0.48}^{+0.49} \mathrm{pb}$, consistent with the SM prediction [21].

Analyses optimized for the $s$-channel search have been performed using the full CDF dataset (up to $9.5 \mathrm{fb}^{-1}$ ) [22]. The lepton+jets analysis forms an NN-discriminant to separate the signal and employ a binned-likelihood technique to extract the ST $s$-channel cross section from the NN output distribution. In the $E_{T}+$ jets analysis the events are classified in the 2-3 jets region with different tagging category (T,TT,TL). The two CDF analyses are combined by taking the products of the likelihood and simultaneously varying the correlated uncertainties. A combined $s$-channel cross section of $\sigma_{s}=1.36_{-0.32}^{+0.37} \mathrm{pb}$ is measured, corresponding to a signal significance of 4.2 stardard deviations [22].

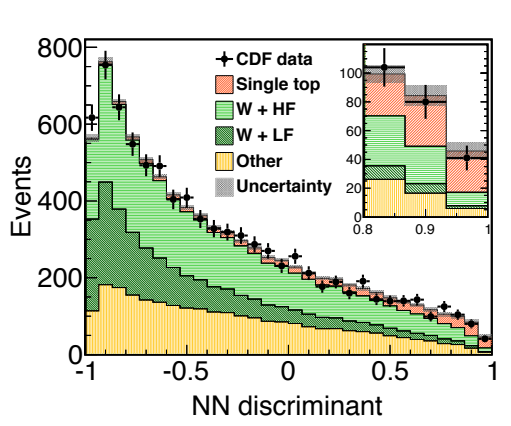

(a)

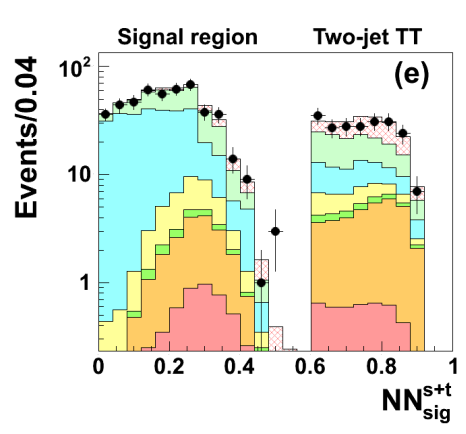

(b)

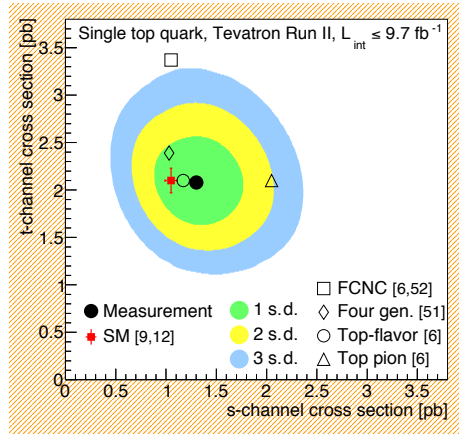

(c)

Figure 3: CDF lepton+jets (a) and $\not_{T}+$ jets (b) final discriminants for the $s+t$ inclusive channel. Posterior density as a function of the $t$ - and $s$-channel single top cross section for the Tevatron $s+t$ combination (c).

\section{Tevatron Single Top Combinations}

\subsection{Single Top $s$-channel observation at Tevatron}

The Tevatron $s$-channel combination is performed using as inputs the CDF lepton+jets (Fig. 4(a)) and $Z_{T}+$ jets (Fig. 4(b))final discriminants and the D0 discriminant (Fig. 2(a)). The combina- 


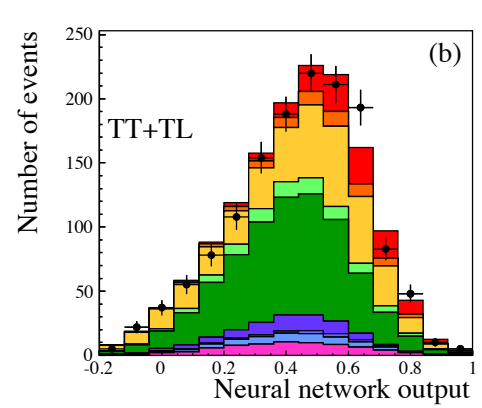

(a)

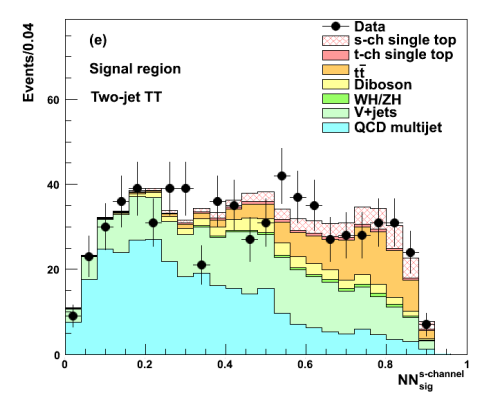

(b)

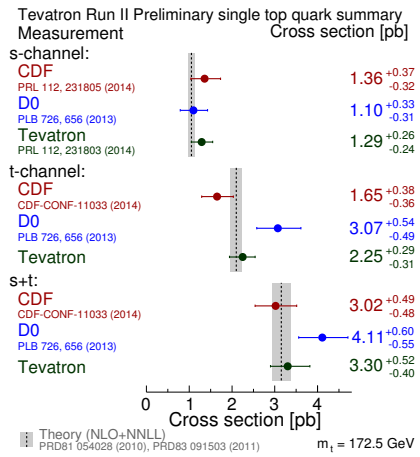

(c)

Figure 4: CDF lepton+jets (a) and $\not_{T}+$ jets (b) final discriminants for the $s$-channel dedicated analyses. Summary of the Tevatron single top quark cross section measurements (c).

tion measures a cross section for the $s$-channel production of $\sigma_{s}=1.29_{-0.24}^{+0.26} \mathrm{pb}$. The measurement corresponds to a significance of 6.3 standard deviations, reporting the first observation of the single top $s$-channel production (which is also the first observation made through a Tevatron combination)[23].

\subsection{Tevatron $s+t$ Combination}

A combination of the CDF and D0 measurements has been recently performed using a total integrated luminosity of up to $9.7 \mathrm{fb}^{-1}$. The combination utilizes the $s$ - and $t$-channel discriminants from $\mathrm{CDF}$ and $\mathrm{D} \emptyset$ single top quark measurements and forms a binned likelihood as a product of all analysis channels in the bins of the multivariate discriminants. The most probable value for the combined $t$-channel cross section is $\sigma_{t}=2.25_{-0.31}^{+0.29} \mathrm{pb}$. The combined $s+t$ cross section is measured without assuming the SM ratio of $\sigma_{s} / \sigma_{t}$ to be $\sigma_{s+t}=3.30_{-0.40}^{+0.52} \mathrm{pb}$. The extracted 2D posterior probability distribution as a function of $\sigma_{t}$ and $\sigma_{s}$ is in Fig.3(c). The CKM matrix element $\left|V_{t b}\right|$ is also directly extracted from the single top cross section measurement without any assumption on the number of quark families or the unitarity of the CKM matrix. The obtained value is $\left|V_{t b}\right|=1.02_{-0.05}^{+0.06}$ and a limit of $\left|V_{t b}\right|>0.92$ at $95 \%$ C.L. is extracted [24].

\section{Search for $W^{\prime}$ bosons decaying to top and bottom quarks with CDF experiment}

Resonances described in Section 1 result in one or more massive charged vector bosons, generically denoted as $W^{\prime}$. A novel search for charged massive resonances decaying to tb quark pair has been recently performed using $9.5 \mathrm{fb}^{-1}$ of CDF data [25]. The search is conducted with $\not_{T}$-based trigger requiring or not presence of a charged lepton in final state. The majority of the background at this stage is QCD production of multijet events which is rejected using a dedicated Neural Network $\left(\mathrm{NN}_{\mathrm{QCD}}\right)$. Events are then classified with respect to the number of jets and the number of b-tags and a final $\mathrm{NN}$-discriminant $\left(\mathrm{NN}_{\mathrm{sig}}\right)$ is used to improve the expected sensitivity in each analysis subsample. A binned likelihood fit is performed over the bins of the $\mathrm{NN}_{\text {sig }}$ distribution to probe a $W^{\prime} \rightarrow t b$ signal in the presence of SM backgrounds. A left-right symmetric BSM model with $W^{\prime}$ bosons of unknown mass and SM weak-coupling to fermions is used as a benchmark model [26]. 


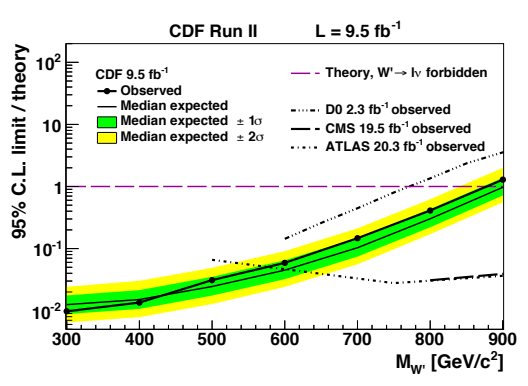

(a)

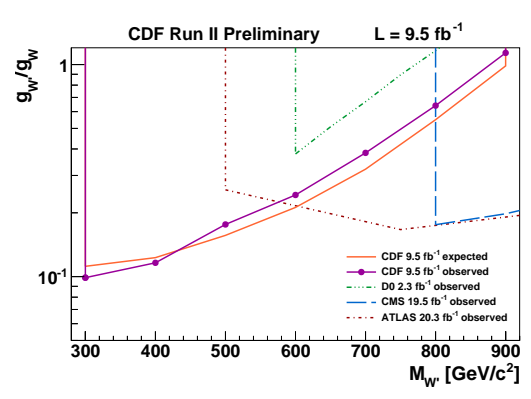

(b)

Figure 5: Observed and expected limits on $\sigma\left(p \bar{p} \rightarrow W^{\prime}\right) \times B\left(W^{\prime} \rightarrow t b\right)$ or a right-handed $W^{\prime}$ boson with SMlike couplings in cases where the leptonic decay mode is forbidden (dashed line) (a). Observed and expected 95\% C.L. upper limits on the coupling strength of a right-handed $W^{\prime}$ boson compared to the SM W-boson coupling in cases where the leptonic decay mode is forbidden (b).

The signal is modeled using Pythia[27]. The observed limits (Fig. 5(a)) are compatible with the expectations calculated assuming that no $W^{\prime} \rightarrow t b$ signal is present in the data. $W^{\prime}$ bosons are escluded for masses less than $860(880) \mathrm{GeV} / \mathrm{c}^{2}$ in cases where $W^{\prime} \rightarrow t b$ decay to leptons are allowed (forbidden). At the time of the conference, this analysis was showing the best exclusion limit for $M_{W^{\prime}} \leq 600 \mathrm{GeV} / \mathrm{c}^{2}$.

\section{Conclusions}

We presented the most recent measurements of the single top quark production and the $\left|\mathrm{V}_{\mathrm{tb}}\right|$ matrix element at the Tevatron, which are summarized in Fig.4(c). The combination between the CDF analyses and the previous D0 analysis on the single top $s$-channel cross section led to the first observation with an uncertainty of $19 \%$ and a significance of 6.3 standard deviations.

All the single top quark measurements at the Tevatron are in agreement with the SM predictions. With the recent finalization of the Tevatron combined $s+t$ production measurement, the Tevatron single top program is mostly complete.

A search for $W^{\prime}$ resonances in the same signature of the single top quark has been also presented. In absence of an evidence of $W^{\prime} \rightarrow t b$ signal in the data, limits have been calculated on a model with a right-handed $W^{\prime}$ boson with SM-like couplings.

\section{References}

[1] F. Abe et al. (CDF Collaboration) Phys. Rev. Lett. 74, (1995) 2626

[2] S. Abachi et al. (D0 Collaboration) Phys. Rev. Lett. 74, (1995) 2632

[3] N.Kidonakis, "Theoretical results for electroweak-boson and single-top production", arXiv:1506.04072.

[4] T. Aaltonen et al. (CDF Collaboration), Phys. Rev. Lett. 103, (2009) 092002.

[5] V. M. Abazov et al. (D0 Collaboration), Phys. Rev. Lett. 103, (2009) 092001;

[6] V. M. Abazov et al. (D0 Collaboration ), Phys. Lett. B, 705, (2011) 313. 
[7] G. Aad et al. (ATLAS Collaboration), Phys.Lett. B717, (2012) 330âĂŞ350.

[8] S. Chatrchyan et al. (CMS Collaboration), JHEP, 1212, (2012) 035.

[9] S. Chatrchyan et al. (CMS Collaboration), Phys.Rev.Lett. 112, (2014) 231802

[10] T.M.P.Tait and C.P.Yuan ,Phys.Rev.D63, (2000) 014018.

[11] T. Aaltonen et al. (CDF Collaboration), Phys. Rev. Lett. 103, 041801 (2009).

[12] V. M. Abazov et al. (D0 Collaboration), Phys. Lett. B 699, 145 (2011).

[13] G. Aad et al. (ATLAS Collaboration), Phys.Lett. B743, 235 (2015).

[14] S. Chatrchyan et al. (CMS Collaboration), J. High Energy Phys. 05 (2014) 108.

[15] E. E. Boos et al., Phys. Atom. Nucl. 69, (2006) 1317.

[16] S. Alioli et al., J. High Energy Phys. 0909, (2009) 111.

[17] V. M. Abazov et al. (D0 Collaboration), Nucl. Instrum.Methods in Phys. Res. Sect. A 620, (2010) 490.

[18] V. M. Abazov et al. (D0 Collaboration), Phys. Rev. Lett. 99, (2007) 191802

[19] V.M. Abazov et al. (D0 collaboration), Phys. Lett. B 726, (2013) 656.

[20] T. Aaltonen et al. (CDF Collaboration) Phys. Rev. Lett. 113, 261804.

[21] T.Aaltonen et al. (CDF collaboration), submitted to Phys.Re.Lett. arXiv:1410.4909.

[22] T. Aaltonen et al. (CDF Collaboration), Phys. Rev. Lett. 112, (2014) 231805.

[23] T. Aaltonen et al. (CDF and D0 Collaboration), Phys. Rev. Lett. 112, (2014) 231803.

[24] T. Aaltonen et al. (CDF and D0 Collaboration), submitted to Phys. Rev. Lett., arXiv:1503.05027.

[25] T. Aaltonen et al. (CDF Collaboration) Phys. Rev. Lett. 115, 061801.

[26] Z. Sullivan, Phys. Rev. D66, (2002) 075011.

[27] T. Sjöstrand, S. Mrenna, and P. Z. Skands, J. High Energy Phys. 05 (2006) 026. 